\title{
Intensivists Perspectives on COVID-19
}

\author{
Sukhyanti Kerai ${ }^{1}$, Kirti N Saxena ${ }^{2}$
}

\begin{abstract}
The coronavirus disease 2019 (COVID-19) pandemic has overwhelmed the healthcare system across the globe. The highly contagious nature of the disease, enormous caseload, and acute hypoxemic respiratory failure associated with the disease has led to a surge in demand for intensive care unit (ICU) beds. As in our country, intensive care facilities are already limited and unevenly distributed, the challenges of providing care to the critically ill are manifold. Furthermore, despite intense research, there is currently no effective treatment against the COVID-19 infection. The ICU mortality rate of COVID-19 is twice compared to other viral pneumonia. Apart from disrupting the physical health, COVID-19 infection has mental health ramifications for ICU patients, such as, depression, insomnia, post-intensive care syndrome. Additionally, the frontline healthcare workers of ICU are at risk of adverse physical and mental consequences directly as the result of providing care to patients. To maintain a sustainable workforce there is an urgent need for the implementation of strategies aimed to reduce the burden of health consequences.

Keywords: COVID-19, Intensive care, ICU.

Indian Journal of Medical Biochemistry (2020): 10.5005/jp-journals-10054-0144
\end{abstract}

\section{INTRODUCTION}

The current coronavirus disease 2019 (COVID-19) pandemic caused by SARS-CoV-2 virus was first reported from China in December 2019 and started spreading throughout the globe like the Spanish flu of 1918. The World Health Organization (WHO) declared the novel coronavirus outbreak (COVID-19) as pandemic on March 11, 2020. In response to this, Lok Nayak Jai Prakash Hospital was declared a dedicated COVID-19 hospital on March 17, 2020. As initial studies from China reported a high incidence of acute respiratory distress syndrome (ARDS) (17-29\%) and critical illness (23-32\%) among hospitalized patients, preparation for an expected increase in demand for critical care services were started. ${ }^{1-3}$ Elective surgical operations were postponed and the non-COVID-19 patients admitted in intensive care unit (ICU) and wards were shifted to other healthcare facilities, so that resources are available for an expected influx of critically ill COVID-19 patients with respiratory failure. The clinical and resource interface between anesthesia and critical care made anesthesiologists frontline warriors against the pandemic, who usually are known to work silently behind the screens in operation theaters. As an intensivist, major challenges encountered including a surge in demand for ICU beds, clinical management of critically ill COVID-19, and risk of infection transmission to healthcare workers (HCWs).

\section{Surge for ICU Beds}

The ongoing pandemic of coronavirus 19 (COVID-19) has put forth an unprecedented demand for ICU services. Globally, more than one-third of patients with confirmed COVID-19 infection were reported to be requiring ICU admission. ${ }^{4}$ The highly contagious nature of the virus, substantial caseload, and predominant respiratory involvement in the disease process leading to a high proportion of patients requiring respiratory support have been the attributable factors for the surge in ICU requirement. These have necessitated a rapid expansion of ICU infrastructure and staffing; with many hospital wards, outpatient departments, and operation rooms being converted to ICUs and non-ICU staff being deployed for ICU services. For low- and middle-income countries with a low limited number of ICU beds, mechanical ventilators,
1,2Department of Anesthesiology and Intensive Care, Maulana Azad Medical College and Associated Hospitals, New Delhi, India

Corresponding Author: Sukhyanti Kerai, Department of Anesthesiology and Intensive Care, Maulana Azad Medical College and Associated Hospitals, New Delhi, India, Phone: +91 9968527122 , e-mail:drsukhi25@gmail.com

How to cite this article: Kerai S, Saxena KN. Intensivists Perspectives on COVID-19. Indian J Med Biochem 2020;24(3):134-136.

Source of support: Nil

Conflict of interest: None

skilled professionals have further intensified the healthcare delivery challenges.

\section{ICU Patients}

The COVID-19 patients with the most severe form as seen in ICU are frequently elderly and with comorbidities. A recent metaanalysis found that in-ICU mortality due to COVID-19 is twice that of other viral pneumonia (pooled ICU mortality of 41.6 vs $22 \%$ ). This mortality has been broadly found to be consistent across the globe. ${ }^{5}$ This may be accounted by more severe cases of COVID19 infection being admitted to ICU, resource constraint due to pandemic leading to difficulty in providing reliable services and to disease process itself.

The treatment of ICU-19 patients with severe disease is challenging as no specific drug is available against the COVID-19 virus. Currently, drugs that either target the intracellular replication and spread of the virus or the cytokine storm inflammation seen in COVID-19 cases during the later stages of the disease are being used for therapy. None of the antiviral drugs have proven efficacy against COVID-19. Antiviral drugs, such as, remdesivir, hydroxychloroquine, and the popular convalescent plasma therapy, have not found to significantly affect clinical outcomes in severe or life-threatening COVID-19 infection. ${ }^{6,7}$ The only drug shown to be beneficial in severe cases is anti-inflammatory agent dexamethasone. Horby et al. in a trial on 2104 patients found dexamethasone reduced 28-day mortality among those receiving mechanical ventilation or 
oxygen. The benefit of dexamethasone was not observed among patients not receiving respiratory support. ${ }^{8}$

As most ICU patients are elderly with comorbidities and the length of time for recovery in ICU survivors on average has been observed to be around 2 to 3 weeks; these patients are at major risk of malnutrition. We particularly faced that many of these patients even before ICU admission had reduced oral intake which harbingered electrolyte and acid-base abnormalities, derangement in kidney functions, and blood sugar abnormalities. Many patients continued to have reduced food intake because of several factors, such as, anorexia, dyspnea, dysgeusia, psychological stress of physical and social isolation, and organizational problems limiting attendance at meals. Severe respiratory infection induced inflammatory syndrome, hypercatabolism, increased energy expenditure in ventilated patients, rand physical immobilization exposing patients to rapid muscle wasting are additional factors for development of malnutrition. ${ }^{9}$ Hence, nutrition is an important element of ICU care and early nutritional care must be integrated in overall therapeutic strategy.

\section{Psychological Issues in ICU Patients}

Along with the brunt of the disease, patients of COVID-19 experience several psychological stressors, such as, difficulty in gaining ICU admission, social and physical isolation, and witnessing the deaths of other patients. The highly contagious nature of COVID-19 has stripped ICU patients of facilities that help them feel emotionally strong. The visit of family members is not allowed, there is less staff coming in and attending, healthcare staffs coming in contact with them are equipped with personal protective equipments (PPEs) rendering them "faceless", there is constant noise and beeps of monitors and ventilators; all of these factors make them psychologically vulnerable. Agitation, altered consciousness, and delirium have been commonly observed in COVID-19 ICU patients. ${ }^{10}$ Even after their recovery, these ICU patients may face the second battle of experiencing "post-intensive care syndrome" (PICS) which manifests as a combination impairment of mental, physical, and cognitive health. ${ }^{11}$

Sahoo et al. reported narrative experience of ICU survivors depicting the mental agony they went through upon being admitted in ICU. These patients had predominant theme of fear of being intubated, dying alone or being away from family, feeling insecure about their families if they die. ${ }^{12}$ Similar to their report, the most common concerns that we have received from our ICU patients is that "What will happen to my wife and children after me, I am not afraid of death"? and "Will I be able to see my family again?". We observed that ICU survivor patients on an average takes 2 or more weeks for recovery. This long period of isolation and loss of human touch frequently makes them emotionally vulnerable.

Therefore, providing psychological support to COVID-19 patients admitted in ICU should be an integral part of ICU management protocols. Healthcare workers should endeavor to encourage and motivate these patients. Virtual methods of communication can be used to connect them to their families. To decrease delirium, sedation in these patients should be minimized, daily sunlight exposure should be ensured, and circadian rhythm should be tried to normalize.

\section{Impact on Frontline Intensivists}

Anesthesiologists and particularly intensivists are at high risk of exposure due to their working environments and exposure to sicker patient groups. Patients with severe COVID-19 requiring
ICU admission have relatively higher respiratory viral shedding. Intensivists spend prolonged periods of time indoor and as the patient in ICU are critical, they need constant attention and care. Furthermore, as the majority of procedures carried out in ICU patients are aerosol generating, there is high risk of infection to HCWs. These include endotracheal intubation, nebulization, suctioning, cardiopulmonary resuscitation, tracheostomy, and bronchoscopy. However, it is reassuring that studies from many countries have reported low infection rate among critical care health workers and an analysis of 106 staff deaths from United Kingdom (UK) found that none were anesthetists, intensive care doctors, or nurses. ${ }^{13}$ It has been suggested those working in ICU and requiring to be close to the airway of critically ill patients may have systematically prepared at protecting themselves against nosocomial infection. Therefore, it is a worthy consideration that meticulous adherence to infection control measures will benefit HCWs working outside the ICU.

The COVID-19 pandemic has had an enormous psychological impact on intensivists. A recent survey reported an overall prevalence of $52 \%$ of burnout in ICU physicians. Factors independently associated with symptoms of anxiety, depression, and burnout were female gender, age, clinician's rating about ethical climate at the workplace, working in a university-affiliated hospital, and living in a city with $>1$ million inhabitants. The COVID-19 ICU experience compared to general non-COVID-19 have significantly found to be emotionally more challenging and have reported to cause significant more intrateam and family conflicts. ${ }^{14}$

During the pandemic, for patients lacking decision-making capacity, tough decisions had to be made without relatives. Intensive care unit clinicians with symptoms of anxiety, depression, and burnout also reported more frequent smoking or taking sleeping pills. National responses to pandemic including lockdown and fear of infection transmission to family members have also worsen the psychological stress of frontline workers.

Difficulties in involving the relatives, the perception of inappropriate care, and suboptimal decision-making have led to moral distress and may be the root cause of clinician burnout.

\section{Conclusion}

The COVID-19 pandemic has created an extraordinary demand for critical care services. As there is no definitive treatment for this disease, the treatment of critically ill patients mainly remains supportive. Taking into account the mental health of admitted patients is of paramount importance as they can continue to affect the quality of life even after ICU survival. The protection of HCWs is also an important consideration to maintain a sustainable workforce against the battle with a pandemic.

\section{References}

1. Yang $X$, Yuhan Yu, Shu XuJ, et al. Clinical course and outcome of critically ill patients with SARS CoV -2 pneumonia in Wuhan, China: a single centred, retrospective, observational study. Lancet Respir Med 2020(5):Available on https://doi.org/10.1016/S2213-2600(20) 30079-5.

2. Chen N, Zhou M, Dong X, et al. Epidemiological and clinical characteristics of 99 cases of 2019 novel coronavirus pneumonia in Wuhan, China: a descriptive study. Lancet 2020;395(10223):507-513. DOI: 10.1016/S0140-6736(20)30211-7.

3. Wang D, Hu B, Hu C, et al. Clinical characteristics of 138 hospitalized patients with 2019 novel coronavirus -infected pneumonia in Wuhan, China. JAMA 2020;323(11):1061-1069. DOI: 10.1001/jama.2020.1585. 
4. Abate SM, Ali SA, Mantfardo B, et al. Rate of intensive care unit admission and outcomes among patient with coronavirus: a systematic review and meta-analysis. PLoS ONE 2020;15(7):e0235653. DOI: 10.1371/journal.pone.0235653.

5. Armstrong RA, Kane AD, Cook TM. Outcomes from intensive care in patients with COVID-19: a systematic review and meta-analysis of observational studies. Anaesthesia 2020(10):Available from: https:// doi.org/10.1111/anae.15201.

6. Li L, Zhang W, Hu Y, et al. Effect of convalescent plasma therapy on time to clinical improvement in patients with severe and life-threatening COVID-19. JAMA 2020;324(5):1-11. DOI: 10.1001/ jama.2020.10044.

7. Wang Y, Zhang D, Du G, et al. Remdesivir in adults with severe COVID19: a randomized, double-blind, placebo-controlled, multicentre trial. Lancet 2020;395(10236):1569-1578. DOI: 10.1016/S01406736(20)31022-9.

8. Horby P, Lim WS, Emberson JR, et al., RECOVERY collaborative group Dexamethasone in hospitalized patients with Covid-19-preliminary report. N Eng J Med 2020. Available from: 10.1056/NEJMoa2021436.
9. Thibault $R$, Seguin $P$, Tamion $F$, et al. Nutrition of COVID-19 patient in the intensive care unit (ICU): a practical guidance. Crit Care 2020;24(1):447. Available from: 10.1186/s13054-020-03159-z.

10. Kotfis K, Roberson SW, Wilson JE, et al. COVID-19: ICU delirium management during SARS-CoV2 pandemic. Crit Care 2020;24(1):176. DOI: 10.1186/s13054-020-02882-x.

11. Jaffri A, Jaffri UA. Post-intensive care syndrome and COVID-19: crisis after a crisis? Heart Lung 2020;49(6):883-884. Available from: https:// dx.doi.org/10.1016\%2Fj.hrtlng.2020.06.006.

12. Sahoo S, Mehra A, Suri V, et al. Lived experiences of COVID-19 intensive care unit survivors. Indian J Psychol Med 2020(4):Available from: https://doi.org/10.1177\%2F0253717620933414.

13. Kursumovic E, Lennane S, Cook TM. Deaths in healthcare workers due to COVID-19: the need for robust data and analysis. Anaesthesia 2020;75(8):989-993. DOI: 10.1111/anae.15116.

14. Azoulay E, De Waele J, Ferrer R, et al. Symptoms of burnout in intensive care specialists facing the COVID-19 outbreak. Ann Intensive Care 2020;10(1):110. Available on: https://dx.doi.org/10.11 86\%2Fs13613-020-00722-3. 